\title{
Les ressources hydroélectriques du Parama brésilien
}

\author{
Hydro-electric resources \\ of the Parana river in Brasil
}

\author{
C. A. de P. Amarante
}

Président du Comité brésilien des Grands Barrages

Le Brésil est un grand pays, ayant une surface de $8500000 \mathrm{~km}^{2}$, et doté d'un immenise réseau fluvial. Ses principaux bassins hydrographiques sont ceux :

- du fleuve Amazonas (3985000 km²),

- du Parana (1237000 $\mathrm{km}^{2}$ ),

- du Tocantins $\left(803000 \mathrm{~km}^{2}\right)$,

- du Sao Francisco $\left(631000 \mathrm{~km}^{2}\right)$,

- de l'Uruguai $\left(178000 \mathrm{~km}^{2}\right)$,

- de l'Atlantique Nord $\left(885000 \mathrm{~km}^{2}\right)$,

- de l'Atlantique Est $\left(569000 \mathrm{~km}^{2}\right)$

- et de l'Atlantique Sud-Est $\left(224000 \mathrm{~km}^{2}\right)$

comme indiqué sur la figure 1.

La connaissance du potentiel hydraulique du Brésil a évolué, progressivement, à mesure que les informations sur les caractéristiques physiques de ses bassins hydrographiques devenaient disponibles, et qu'étaient réalisées des études (dans les bureaux et sur le terrain) permettant de définir la répartition des chutes le long des rivières composant ces bassins.

Jusqu'alors, on ne possédait aucun inventaire complet du potentiel hydraulique du Brésil, mais on a cherché à l'évaluer en additionnant les résultats obtenus sur le terrain et diverses estimations réalisées en bureau, concernant les zones du pays non encore inventoriées.

Un premier travail systématique d'étude du potentiel hydraulique réalisé sur le terrain s'est limité géographiquement à la région Sud-Est du pays, en raison de sa proximité des grands centres de consommation d'énergie électrique - les régions métropolitaines de Sao Paulo et de Rio de Janeiro.

Cette étude couvrait la majeure partie du bassin du Parana brésilien, à l'exclusion de l'Iguaçu inventorié plus tard, mais comprenait d'autres bassins comme ceux du Doce, du Paraiba et une partie du S. Francisco.

Les études réalisées entre 1962 et 1966 ont constitué une initiative pionnière pour le pays et sans précédent dans le monde entier. La méthodologie adoptée a servi de base pour toutes les études postérieures.

La région étudiée comprenait $1000000 \mathrm{~km}^{2}$ soit l'équivalent des surfaces additionnées de la France, de l'Espagne et du Portugal ; vingt huit mille kilomètres de fleuves ont été parcourus et plus de 510 sites de barrages ont été examinés.

Le plan d'électrification, élaboré en 1966, a consisté en un programme de longue durée, s'étendant jusqu'à 1980 , et comprenant aussi l'examen d'autres sources d'énergie primaires comme le charbon, les combustibles liquides et les centrales nucléaires.

A l'époque des études, le système électrique de la région Sud-Est était capable de fournir une énergie annuelle de $2540 \overline{\mathrm{MW}}{ }^{1}$ ) ou 22,25 TWh et de satisfaire à une demande d'énergie moyenne de $4096 \mathrm{MWh} / \mathrm{h}$. Les perspectives énergétiques pour les quinze années à venir pour 1980 étaient les suivantes :

- Evaluation haute $9962 \overline{\mathrm{MW}}$ ou 87,3 TWh/an

- Evaluation basse $7609 \overline{\mathrm{MW}}$ ou 66,6 TWh/an

- Réalisé $8778 \overline{\mathrm{MW}}$ ou $76,9 \mathrm{TWh} / \mathrm{an}$

Les réalisations ont respecté avec beaucoup de précision les estimations faites 15 ans auparavant.

Dans le cas brésilien, il faut souligner l'importance de l'énergie hydroélectrique, par rapport à l'énergie thermoélectrique, celle-ci se présente normalement comme complémentaire, et est maintenue à un niveau minime de production uniquement pour satisfaire les demandes de pointe et de basse hydraulicité.

L'existence d'un potentiel hydroélectrique très important et économique à explorer d'une part, l'exigence d'investissements élevés pour l'édification des

(1) $1 \overrightarrow{\mathrm{MW}}=8760 \mathrm{MWh} / \mathrm{an}=8,76 \mathrm{GWh} / \mathrm{an}=8,76 \cdot 10^{-3}$ TWh/an.

LA HOUILLE BLANCHE/N ${ }^{\circ}$ 5/6-1982 
centrales thermiques à charbon et nucléaires, d'autre part, font que la tendance naturelle du système électrique brésilien est de poursuivre essentiellement la production hydroélectrique au moins jusqu'à l'an 2000.

Le potentiel hydroélectrique du Brésil en termes d'énergie garantie et moyenne est mentionné sur le Tableau I, en fonction des principaux bassins hydrographiques du pays.

\section{BASSINS HYDROGRAPHIQUES DU BRESIL}

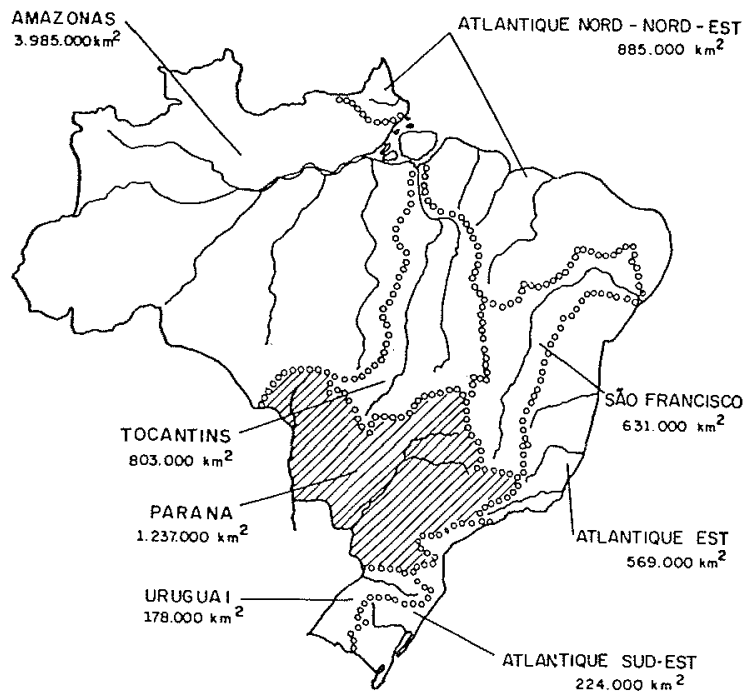

Figure 1 - Bassins hydrographiques du Brésil.
On peut voir sur ce Tableau I que le bassin du Parana représente le second potentiel hydroélectrique du Brésil, dépassé seulement par celui du bassin de l'Amazonas.

Le potentiel du Parana correspond à presque $27 \% \mathrm{du}$ potentiel total du Brésil et son importance est soulignée par sa situation au cceur de la région la plus développée, la plus peuplée et la plus industrialisée du pays.

On peut également voir qu'à l'horizon 1990, le potentiel énergétique aménagé pour l'ensemble du pays est estimé à $33 \widehat{\mathrm{GW}}$ (ou $289 \mathrm{TWh} / \mathrm{an}$ ) et que le bassin du Parana, à lui seul, représentera 19,8 $\overline{\mathrm{GW}}$ (ou $173 \mathrm{TWh} / \mathrm{an}$ ) soit près de $60 \%$ de la puissance globale, le plaçant très largement en tête des bassins producteurs d'énergie hydroélectrique et ceci pour une longue période à venir.

On peut ainsi en déduire eu égard à sa situation, l'importance fondamentale du potentiel hydroélectrique du bassin du fleuve Parana sur le développement du Brésil.

\section{Le bassin du Parana}

Le fleuve Parana a pour origine, le confluent des fleuves Paranaiba et Grande, il coule initialement en territoire brésilien en direction du Sud-Ouest, puis il forme la frontière entre le Brésil et le Paraguai sur $200 \mathrm{~km}$, suit la frontière entre le Paraguai et l'Argentine et coule ensuite en territoire argentin jusqu'au fleuve de la Plate (Rio da Prata).

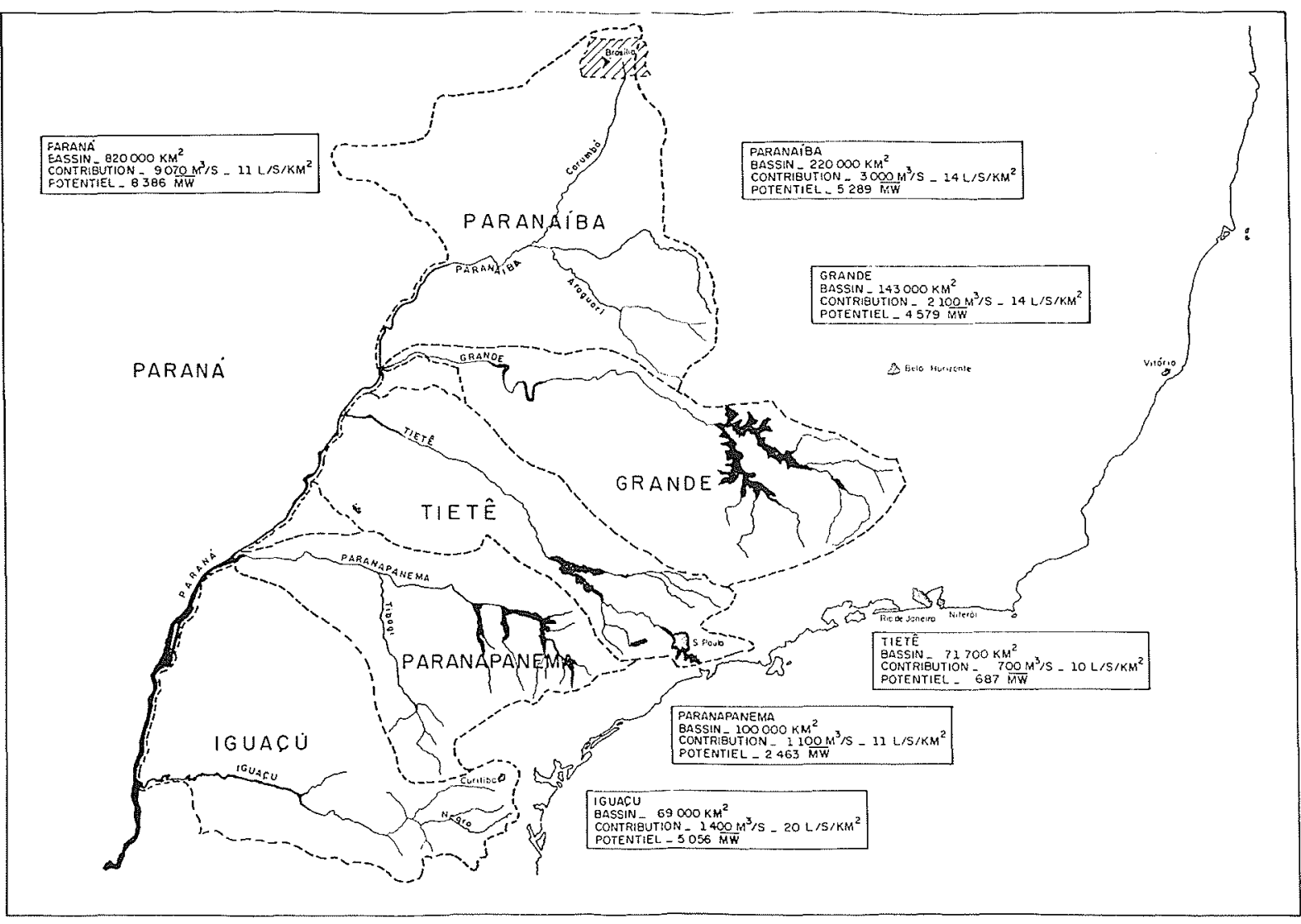

Figure 2 - Bassin du Parana et des principaux sous-bassins. 


\begin{tabular}{|c|c|c|c|c|c|c|}
\hline \multicolumn{7}{|c|}{ Tableau I. - Potentiel énergétique du Brésil } \\
\hline Bassin & $\begin{array}{r}\text { Ener } \\
\text { garan } \\
\text { en }\end{array}$ & $\begin{array}{l}\text { gle } \\
\text { titie } \\
\overline{\mathrm{MW}}\end{array}$ & $\begin{array}{c}\text { Energie } \\
\text { aménagée } \\
\text { jusqu'à } \\
1990 \\
\text { GW }\end{array}$ & $\begin{array}{c}\text { Energie } \\
\text { disponible } \\
\text { après } \\
1990 \\
\overline{G W}\end{array}$ & $\begin{array}{l}\text { Energie } \\
\text { Moyenne } \\
\text { MWh/h }\end{array}$ & $\begin{array}{c}\text { Surface } \\
\mathrm{km}^{2}\end{array}$ \\
\hline $\begin{array}{l}\text { Amazonas } \\
\text { Tocantins } \\
\text { Atlantique N/NE } \\
\text { Sao Francisco } \\
\text { Atlantique Est } \\
\text { Parana } \\
\text { Uruguai } \\
\text { Atlantique Sud-Est }\end{array}$ & $\begin{array}{r}317,11 \\
111,25 \\
7,88 \\
80,59 \\
57,81 \\
249,66 \\
64,82 \\
43,80\end{array}$ & $\begin{array}{r}36,2 \\
12,7 \\
0,9 \\
9,2 \\
6,6 \\
28,5 \\
7,4 \\
5\end{array}$ & $\begin{array}{r}0,1 \\
4,3 \\
0,1 \\
5,2 \\
1,3 \\
19,8 \\
0,8 \\
1,3\end{array}$ & $\begin{array}{r}36,1 \\
8,4 \\
0,8 \\
4,0 \\
5,3 \\
8,7 \\
6,6 \\
3,7\end{array}$ & $\begin{array}{r}52,4 \\
15,2 \\
1,3 \\
11,8 \\
8,9 \\
34,3 \\
7,2 \\
5,3\end{array}$ & $\begin{array}{r}3985000 \\
803000 \\
885000 \\
631000 \\
569000 \\
1237000 \\
178000 \\
224000\end{array}$ \\
\hline Total & 932 & 106,5 & 32,9 & 73,6 & 136,4 & 8512000 \\
\hline
\end{tabular}

Le bassin entier comprend 3 millions de $\mathrm{km}^{2}$, parmi lesquels $1237000 \mathrm{~km}^{2}$ sont situés au Brésil en $\mathrm{y}$ incluant le sous-bassin du Paraguai.

La longueur totale du fleuve Parana, y compris les affluents Grande et Paranaiba, est de $4000 \mathrm{~km}$. Le Parana est l'un des sept plus grands fleuves du monde, à cause de son débit et de l'étendue de son bassin. Les limites supérieures de ce bassin se situent, en altitude moyenne à environ $1000 \mathrm{~m}$, alors que le niveau moyen à Itaipu n'est, que de $100 \mathrm{~m}$.

Dans la partie septentrionale du bassin le climat est tropical, avec des saisons pluvieuses en été (Décembre à Mai) et sèches en hiver (Juillet à Novembre). En direction du Sud, le climat est plus tempéré avec des étés chauds et des hivers froids, mais les précipitations sont plus uniformément réparties sur toute l'année. La précipitation moyenne annuelle dans le bassin du Parana est de $1400 \mathrm{~mm}$.

Des deux fleuves qui forment le Parana, on peut dire :

- que le Grande se développe tout au long de ses $1300 \mathrm{~km}$ dans un bassin dont la surface est de 143000

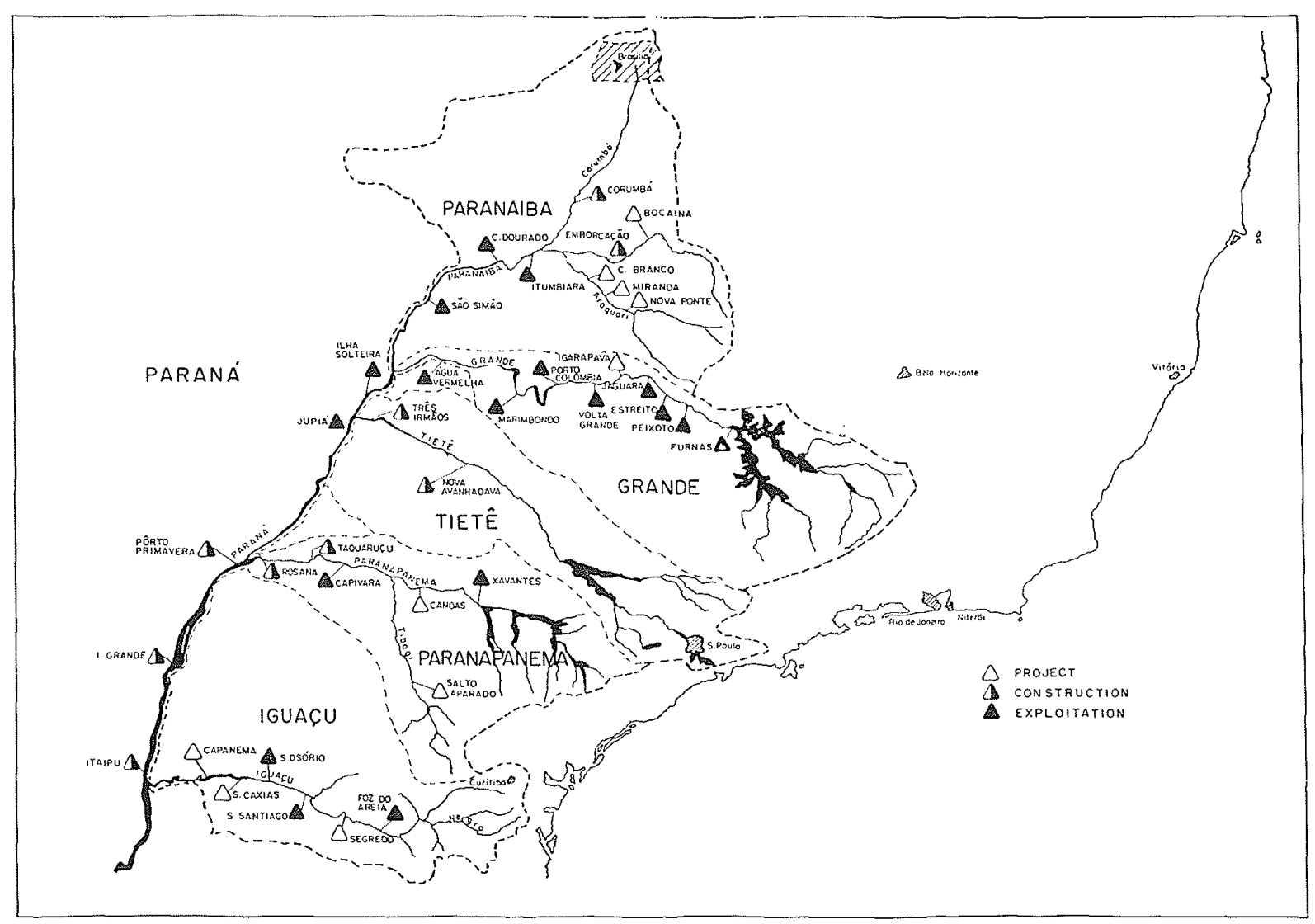

Figure 3 - Bassin du Parana Aménagements de plus de $100 \overline{\mathrm{MW}}$. 
$\mathrm{km}^{2}$ avec urr débit de $2100 \mathrm{~m}^{3} / \mathrm{s}$, (ou près de 14 $\mathrm{l} / \mathrm{s} / \mathrm{km}^{2}$ ),

- tandis que le Paranaiba qui est formé par plusieurs affluents est situé dans un bassin hydrographique de $222000 \mathrm{~km}^{2}$ et assure un débit moyen de $3000 \mathrm{~m}^{3} / \mathrm{s}$. (ou de l'ordre de $14 \mathrm{l} / \mathrm{s} / \mathrm{km}^{2}$ ).

Les principaux affluents sur la rive gauche du Parana brésilien sont : le Tietê, le Paranapanema et l'Iguaçu.

Le Tietê a une longueur de près de $1050 \mathrm{~km}$ et la surface de son bassin hydrographique est de $71700 \mathrm{~km}^{2}$,
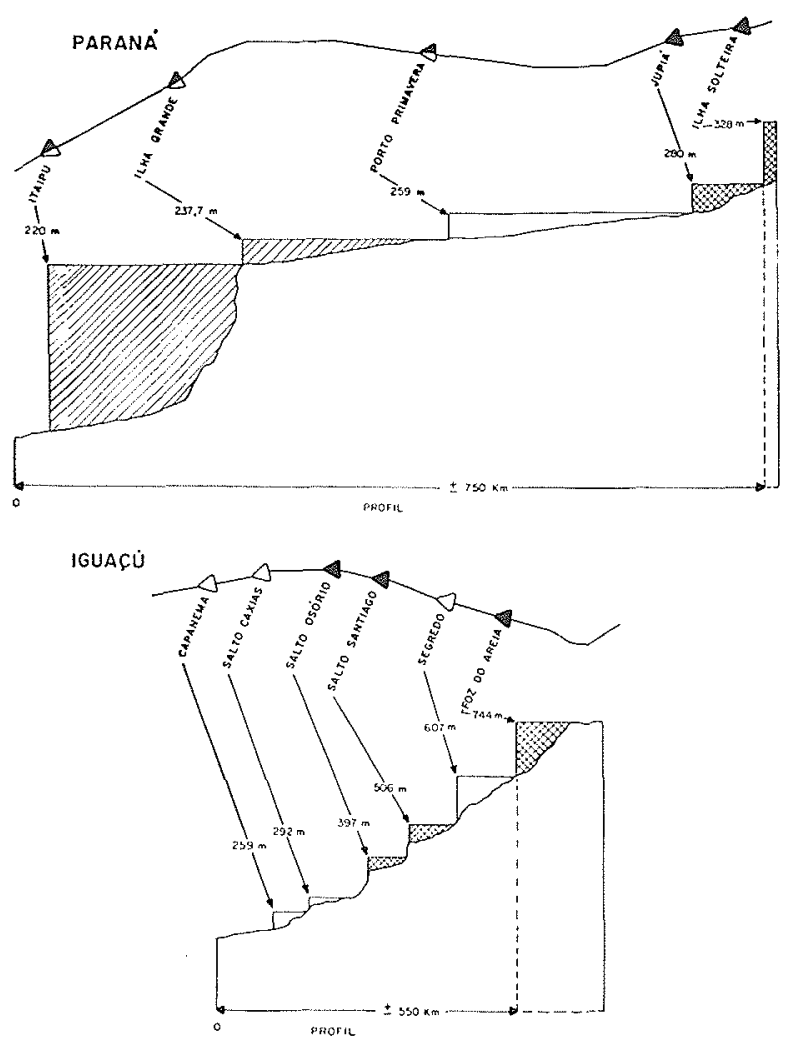

avec une contribution moyenne de l'ordre de $700 \mathrm{~m}^{3} / \mathrm{s}$ (ou de $101 / \mathrm{s} / \mathrm{km}^{2}$ ).

Le Paranapanema a une longueur de près de $600 \mathrm{~km}$, avec un bassin hydrographique de $100000 \mathrm{~km}^{2}$ et un débit moyen de $1100 \mathrm{~m}^{3} / \mathrm{s}$ (ou $111 / \mathrm{s} / \mathrm{km}^{2}$ ).

L'Iguaçu rejoint de Parana au point où convergent les territoires du Brésil, du Paraguai et de l'Argentine; il a $1300 \mathrm{~km}$ de long, draîne une surface de $69000 \mathrm{~km}^{2}$ et sa contribution moyenne est de $1400 \mathrm{~m}^{3} / \mathrm{s}$ (ou $\left.20 \mathrm{l} / \mathrm{s} / \mathrm{km}^{2}\right)$. Les "Cataratas do Iguaçu" très renommées, avec $82 \mathrm{~m}$ de haut, sont situées à $23 \mathrm{~km}$ du confluent de l'Iguaçu et du Parana.

Le débit moyen annuel du Parana à Itaipu, sur une période de 40 années, est de $9070 \mathrm{~m}^{3} / \mathrm{s}$, ce qui correspont à $11,11 / \mathrm{s} / \mathrm{km}^{2}$.

La géologie d'une grande partie de ce bassin du Sud-Est et du Sud du Brésil, est uniforme et consiste en un épais épanchement basaltique, pratiquement horizontal, uniformément intercalé avec des couches de "Brécia". Des terrains résiduels d'argile ou d'argile sablonneux couvrent les rives du Parana et de ses affluents.

Cette caractéristique géologique a conduit à édifier un type d'aménagement commun à ce bassin et comprenant une partie centrale en béton et des flancs en terre compactée.

Le potentiel hydroélectrique total du Parana brésilien et sa répartition par sous-bassins principaux sont donnés dans le tableau II :

Ce potentiel est réparti sur un grand nombre de sites dont :

- 73 en exploitation :

- 11 en construction,

- et 360 disponibles identifiés,

soit un total de 444 sites de barrages pour le bassin.

Les principaux aménagements de potentiel énergétique supérieur à $100 \overline{\mathrm{MW}}$, selon les différents sousbassins sont mentionnés dans le tableau III .

\begin{tabular}{|c|c|c|c|c|}
\hline \multicolumn{5}{|c|}{ Tableau II. - Potentiel hydroélectrique du Parana Brésilien - $\overline{\mathrm{MW}}$} \\
\hline A) Sous Bassin & En exploitation & En construction & Disponible & Total \\
\hline $\begin{array}{l}\text { Paranaiba } \\
\text { Grande } \\
\text { Paraná } \\
\text { Tietê } \\
\text { Paranapanema } \\
\text { Iguaçu }\end{array}$ & $\begin{array}{r}2463 \\
3310 \\
2358 \\
240 \\
602 \\
1972\end{array}$ & $\begin{array}{r}655 \\
58 \\
2028 \\
268 \\
335 \\
-\end{array}$ & $\begin{array}{r}2171 \\
1211 \\
0 \\
179 \\
1526 \\
3084\end{array}$ & $\begin{array}{r}5289 \\
4579 \\
4386 \\
687 \\
2463 \\
5056\end{array}$ \\
\hline \multicolumn{5}{|l|}{ B) Petits Bassins } \\
\hline $\begin{array}{l}\text { Ivai-Piquiri } \\
\text { Paraguai } \\
\text { Divers }\end{array}$ & $\begin{array}{r}7 \\
7 \\
13\end{array}$ & $\begin{array}{l}- \\
- \\
0\end{array}$ & $\begin{array}{r}1154 \\
641 \\
472\end{array}$ & $\begin{array}{r}1161 \\
648 \\
485\end{array}$ \\
\hline Total national & 10972 & 3344 & 10438 & 24754 \\
\hline $\begin{array}{l}\text { C) Production internatio- } \\
\text { comprenant la partie } \\
\text { brésilienne }\end{array}$ & & 3994 & 600 & 4594 \\
\hline Total Général & 10972 & 7338 & 11038 & 29348 \\
\hline
\end{tabular}




\begin{tabular}{|c|c|c|c|c|c|c|c|c|}
\hline \multicolumn{9}{|c|}{ Tableau III. - Aménagements avec énergie garantie supérieure à $100 \overline{\mathrm{MW}}$} \\
\hline Sous-bassin & $\begin{array}{l}\text { Hauteur } \\
\text { de } \\
\text { Chute }\end{array}$ & \multicolumn{5}{|c|}{ En exploitation ou construction } & \multicolumn{2}{|c|}{ Disponible } \\
\hline \multirow{3}{*}{ Grande } & & & $\overline{M W}$ & MW & $E$ & C & \multirow[b]{2}{*}{ Igarapava } & $\overline{\mathrm{MW}}$ \\
\hline & $\begin{array}{r}100 \\
40 \\
68 \\
46 \\
17 \\
27 \\
\\
20 \\
63 \\
55\end{array}$ & $\begin{array}{l}\text { Furnas } \\
\text { Peixoto } \\
\text { Estreito } \\
\text { Jaguara } \\
\text { Volta } \\
\text { Grande } \\
\text { P. Colombia } \\
\text { Marimbondo } \\
\text { A. Vermelha }\end{array}$ & $\begin{array}{l}522 \\
256 \\
437 \\
314 \\
\\
\\
205 \\
189 \\
633 \\
671\end{array}$ & $\begin{array}{r}1216 \\
477 \\
1048 \\
425 \\
\\
\\
380 \\
319 \\
1444 \\
1380\end{array}$ & $\begin{array}{l}x \\
x \\
x \\
x \\
\\
x \\
x \\
x \\
x \\
x\end{array}$ & & & 121 \\
\hline & & Total & 3227 & 6690 & & & & 121 \\
\hline \multirow[t]{2}{*}{ Iguaçu } & $\begin{array}{r}137 \\
101 \\
59 \\
109 \\
72 \\
66 \\
53\end{array}$ & $\begin{array}{l}\text { F. Areia } \\
\text { S. Santiago } \\
\text { S. Osório }\end{array}$ & $\begin{array}{l}565 \\
\\
831 \\
573\end{array}$ & $\begin{array}{l}2511 \\
2000 \\
1053\end{array}$ & $\begin{array}{l}x \\
x\end{array}$ & & $\begin{array}{l}\text { Segredo } \\
\text { Jacu }\end{array}$ & $\begin{array}{l}697 \\
101 \\
\\
\\
665 \\
480\end{array}$ \\
\hline & & Total & 1969 & 5564 & & & & 1943 \\
\hline \multirow[t]{2}{*}{ Paraná } & $\begin{array}{r}48 \\
21 \\
20 \\
19 \\
120\end{array}$ & $\begin{array}{l}\text { I. Solteira } \\
\text { Jupiá } \\
\text { P. Primavera } \\
\text { 1. Grande } \\
\text { Itaipu }\end{array}$ & $\begin{array}{r}1531 \\
827 \\
891 \\
1137 \\
3994\end{array}$ & $\begin{array}{l}3230 \\
1411 \\
1800 \\
2000 \\
6301\end{array}$ & $\begin{array}{l}x \\
x\end{array}$ & $\begin{array}{l}x \\
x \\
x\end{array}$ & & \\
\hline & & Total & 8380 & 14742 & $\mathrm{x}$ & $x$ & & 0 \\
\hline \multirow[t]{2}{*}{ Paranaíba } & $\begin{array}{r}76 \\
137 \\
104 \\
81 \\
99 \\
100 \\
85 \\
29 \\
73\end{array}$ & $\begin{array}{l}\text { Emborcação } \\
\text { Corumbá } \\
\text { Itumbiara } \\
\text { C. Dourada } \\
\text { S. Simão }\end{array}$ & $\begin{array}{r}244 \\
915 \\
342 \\
1189\end{array}$ & $\begin{array}{r}1000 \\
\\
\\
\\
500 \\
2100 \\
416 \\
1613\end{array}$ & $\begin{array}{l}x \\
x \\
x\end{array}$ & $x$ & $\begin{array}{l}\text { Bocaina } \\
\text { N. Ponte } \\
\text { Miranda } \\
\text { C. Branco }\end{array}$ & $\begin{array}{l}108 \\
\\
214 \\
123 \\
218\end{array}$ \\
\hline & & Total & 3101 & 5629 & $\mathrm{x}$ & $x$ & & 663 \\
\hline \multirow[t]{2}{*}{ Paranapanema } & $\begin{array}{l}76 \\
32 \\
70 \\
50 \\
26 \\
19\end{array}$ & $\begin{array}{l}\text { Xavantes } \\
\text { Capivara } \\
\text { Taquaruçu } \\
\text { Rosana }\end{array}$ & $\begin{array}{l}170 \\
\\
323 \\
205 \\
130\end{array}$ & $\begin{array}{l}414 \\
\\
640 \\
500 \\
320\end{array}$ & $x$ & $\begin{array}{l}x \\
x\end{array}$ & $\begin{array}{l}\text { Canoas } \\
\text { S. Aparado }\end{array}$ & $\begin{array}{l}131 \\
115\end{array}$ \\
\hline & & Total & 828 & 1874 & & & & 246 \\
\hline $\begin{array}{l}\text { Tietê } \\
\text { Divers }\end{array}$ & $\begin{array}{r}30 \\
48 \\
\\
56 \\
200 \\
70\end{array}$ & $\begin{array}{l}\text { Navanhandava } \\
\text { Três Irmãos }\end{array}$ & $\begin{array}{l}109 \\
159\end{array}$ & $\begin{array}{l}300 \\
640\end{array}$ & & $\begin{array}{l}x \\
x\end{array}$ & $\begin{array}{l}\text { Ivatuba } \\
\text { Corrente } \\
\text { S. Apertados }\end{array}$ & $\begin{array}{l}178 \\
111 \\
212\end{array}$ \\
\hline
\end{tabular}



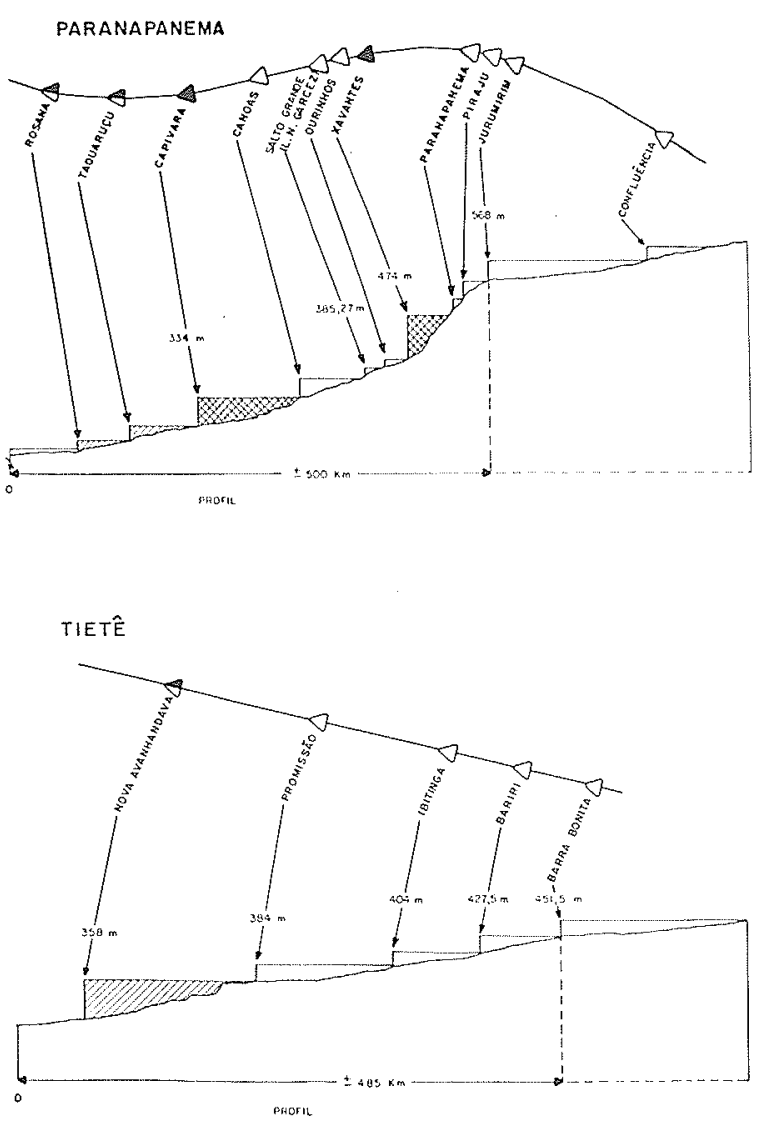

GRANDE

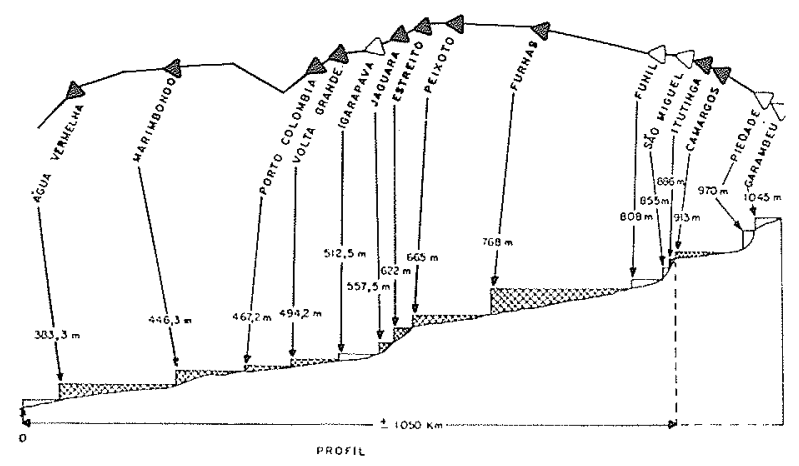

PARANAIBBA

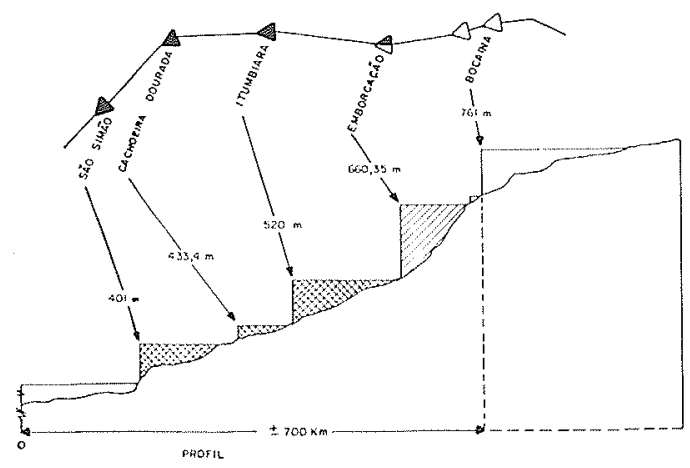

Ces principaux aménagements peuvent être implantés aux positions optimales, en plan et en profil, les plus favorables à l'utilisation de chacun des principaux fleuves du bassin du Parana.

\section{I - Aménagements opérationnels d'une capacité énergétique supérieure à $400 \overrightarrow{\mathrm{MW}}$.}

Une description résumée des principaux aménagements d'une capacité énergétique supérieure à $400 \overline{\mathrm{MW}}$ (ou 3,5 TWh/an) est faite ci-dessous :

\section{Furnas}

Cet aménagement est le principal réservoir du Grande - d'une capacité de 15 billions de $\mathrm{m}^{3}$ - intéres* sant par la régularisation tous les projets en aval.

Il consiste en un barrage d'enrochement à noyaux d'argile, de près de $120 \mathrm{~m}$ de hauteur ayant un volume de $9,5 \times 10^{6} \mathrm{~m}^{3}$.

Furnas possède une installation de $1200 \mathrm{MW}$ de puissance nominale et une capacité ferme de $522 \overline{\mathrm{MW}}$ (ou 4,6 TWh/an). Son exploitation a commencé en 1963.

\section{Marimbondo}

Situé sur le Grande, il consiste en un barrage central en béton avec meneaux de terre compactée. Il a une puissance nominale de $1440 \mathrm{MW}$ et une capacité ferme de $633 \overline{\mathrm{MW}}$ (ou 5,5 TWh/an). Le volume de béton est de $1,2 \times 10^{6} \mathrm{~m}^{3}$ et celui de terre $14,4 \times 10^{6} \mathrm{~m}^{3}$.

Marimbondo est entré en opération en 1975 .

\section{Agua Vermelha}

Il s'agit de l'usine la plus en aval sur le Rio Grande, un peu en amont du confluent avec le Paranaiba, où les deux cours d'eau forment le Parana. L'usine vient d'être terminée et sa puissance finale installée est de $1380 \mathrm{MW}$ ( 6 groupes de $230 \mathrm{MW}$ ). Son barrage est en terre avec une hauteur de $57 \mathrm{~m}$, une longueur de $3920 \mathrm{~m}$ et un volume de $22485000 \mathrm{~m}^{3}$.

\section{Ilha Solteira}

Situé sur le Parana, entre les états de Sao Paulo et du Mato Grosso do Sul, cet aménagement constitue, avec sa puissance installée de $3200 \mathrm{MW}$ (20 unités de $160 \mathrm{MW}$ ) la plus grande usine hydroélectrique en service au Brésil.

Son barrage du type "poids" est en terre et en béton. Il a $74 \mathrm{~m}$ de hauteur, $6160 \mathrm{~m}$ de longueur, et son volume comprend $18842000 \mathrm{~m}^{3}$ de terre et $3676000 \mathrm{~m}^{3}$ de béton.

\section{Jupia}

C'est l'aménagement le plus en amont sur le Parana, en exploitation actuellement.

Il consiste en un barrage - poids, comprenant $7 \times 10^{6} \mathrm{~m}^{3}$ de terre compacte et $1,4 \times 10^{6} \mathrm{~m}^{3}$ de béton. 
C'est un projet de chute basse $(20 \mathrm{~m})$ qui a été mis en service en 1962. Son installation est de $1200 \mathrm{MW}$ et sa capacité énergétique de $827 \overline{\mathrm{MW}}$ (ou 7,24 TWh/an).

\section{Itumbiara}

Construite sur le Paranaiba, entre les états de Minas Gerais et de Goiás, cette usine aura une puissance finale installée de $2100 \mathrm{MW}$ (6 groupes de $350 \mathrm{MW}$ ) ; son premier groupe a commencé à fonctionner en avril 1980.

Avec $35500000 \mathrm{~m}^{3}$ de matériaux compactés, une hauteur de $106 \mathrm{~m}$ et une longueur de $6260 \mathrm{~m}$, ce barrage en terre est l'un des plus grands du Brésil.

\section{Sao Simão}

Ce projet, le plus en aval du Bassin du Paranaíba, est constitué par un barrage central en béton avec flancs de terre et enrochements. Comprenant $12,8 \times 10^{6} \mathrm{~m}^{3}$ de terre, $9.5 \times 10^{6} \mathrm{~m}^{3}$ d'enrochement et $375 \times 10^{3} \mathrm{~m}^{3}$ de béton.

S. Simao a une installation de $1632 \mathrm{MW}$ et une capacité de $1189 \overline{\mathrm{MW}}$ (ou 10,4 TWh/an).

\section{Foz do Areia}

Cet aménagement hydroélectrique, le plus grand en amont sur l'Iguaçu joue un rôle important dans la régularisation du débit pour les utilisations en aval. La centrale aura une puissance installée de $2511 \mathrm{MW}$ (6 groupes de 418,5 MW). Le premier groupe a commencé à produire en Septembre 1980.

Avec ses $160 \mathrm{~m}$ de hauteur, ses $850 \mathrm{~m}$ de longueur et son volume de $13000000 \mathrm{~m}^{3}$, le barrage d'enrochement avec masque amont en béton, se place parmi les plus hauts du monde de ce type.

\section{Salto Santiago}

Situé sur l'Ignaçu, cette usine aura une puissance installée de $2000 \mathrm{MW}$ (6 groupes de $333 \mathrm{MW}$ ); le premier groupe est entré en opération en Décembre 1980.

Outre le barrage en enrochement avec noyau d'argile qui ferme le lit principal du fleuve, il a fallu construire trois digues pour la fermeture des cols existants. Le barrage, du type enrochement avec noyau d'argile, a une longueur totale de 1400 mètres, une hauteur de $80 \mathrm{~m}$ et un volume total de $9860000 \mathrm{~m}^{3}$.

\section{II - Aménagements en cours de construction ou en projet}

Parmi les projets en construction du bassin du Parana, d'une capacité supérieure à $400 \overline{\mathrm{MW}}$, on rencontre les sites suivants :

\section{Emborcacao}

Située sur le Paranaibá qui, avec le Rio Grande, forme le Parana, cette usine d'une puissance finale installée de $1000 \mathrm{MW}$ (4 unités de $250 \mathrm{MW}$ ), a son premier groupe en service depuis Juillet 1982 .

Le barrage, en voie d'achèvement, est en enrochement tout-venant avec noyau d'argile ; il a $158 \mathrm{~m}$ de hauteur, $1500 \mathrm{~m}$ de longueur et un volume de $25000000 \mathrm{~m}^{3}$.

\section{Porto Primavera}

Situé sur le cours principal du Parana en aval de la centrale de Jupia, cet aménagement est prévu pour une puissance installée de $1800 \mathrm{MW}$ et une puissance garantie de $891 \overline{\mathrm{MW}}$ (ou 7,80 TWh/an). La chute a une hauteur de $20 \mathrm{~m}$ et le barrage est en terre compactée avec une partie centrale en béton.

Sa construction a commencé.

\section{Grande}

Situé sur le cours principal du Parana juste en amont du réservoir de Itaipu, ce barrage en terre compactée avec partie centrale en béton est prévu pour une hauteur de chute de $20 \mathrm{~m}$ et une installation d'une puissance nominale de $2000 \mathrm{MW}$.

Sa construction a commencé.

\section{Itaipu}

C'est le plus grand aménagement du bassin du Parana, et présentement, le plus grand projet hydroélectrique en construction dans le monde. Itaipu est situé sur le fleuve Parana, entre le Brésil et le Paraguai à $20 \mathrm{~km}$ au Nord de la frontière entre le Brésil, le Paraguai et l'Argentine.

\section{III - Centrale d'Itaipu}

\section{a) Disposition générale}

Le projet consiste en une série de barrages de types divers avec couronnements à la cote 225 . La centrale est située au pied du barrage principal, transversalement au fleuve. Le déversoir reste localisé sur le plateau de la rive droite.

La puissance nominale de la centrale est de $12600 \mathrm{MW}$, divisée en 18 groupes équipés de turbines type Francis, de $715 \mathrm{MW}$, dont 9 générateurs $60 \mathrm{~Hz}$ de $737 \mathrm{MVA}$ et 9 générateurs $50 \mathrm{~Hz}$ de $823,6 \mathrm{MVA}$.

Les unités de $60 \mathrm{~Hz}$ alimenteront le système brésilien de distribution en courant alternatif après transformation du $500 \mathrm{kV}$ en $750 \mathrm{kV}$.

Les unités de $50 \mathrm{~Hz}$ alimenteront le système électrique paraguayen en $500 \mathrm{kV}$ et $220 \mathrm{kV}$. Lorsqu'il y aura un excès de puissance en $50 \mathrm{~Hz}$, celle-ci sera transférée au Brésil sous forme de courant continu $600 \mathrm{kV}$.

Il est prévu que cet aménagement opèrera essentiellement comme usine de base, au fil de l'eau, avec le niveau d'eau au réservoir maintenu approximativement constant. 


\section{b) Réservoir}

Au niveau d'eau maximum normal (côte $220 \mathrm{~m}$ ), le réservoir aura une capacité brute de 29 billions de mètres cubes et couvrira une surface de $1350 \mathrm{~km}^{2}$.

\section{c) Dérivation provisoire du fleuve}

Pour la construction du barrage principal et de la centrale, le Parana a été détourné de son cours par un canal latéral creusé dans la rive gauche, ce canal a les dimensions suivantes : $2000 \mathrm{~m}$ de longueur, $150 \mathrm{~m}$ de largeur au fond, et $90 \mathrm{~m}$ de profondeur maximum. Pour réaliser ce canal il a été nécessaire d'extraire 22500000 $\mathrm{m}^{3}$ de roches et de terres, matériaux dont la plus grande partie a été réutilisée pour la construction du barrage. Pour la fermeture de la dérivation provisoire, un ouvrage spécial en béton a été construit avec 12 pertuis permettant un débit maximal de $35000 \mathrm{~m}^{3} / \mathrm{s}$ sans déversement des batardeaux dans le lit du fleuve. La fermeture de la dérivation et la mise en eau du réservoir ont eu lieu en Octobre 1982.

\section{d) Barrage principal}

Le barrage principal, du type poids en béton, a $1064 \mathrm{~m}$ de longueur et une hauteur maximale de $196 \mathrm{~m}$ au-dessus du point le plus bas de la fondation. Le volume de béton s'élève à $5160000 \mathrm{~m}^{3}$. Le barrage est établi sur du basalte sain.

\section{e) Barrages latéraux}

Sur la rive droite, le barrage principal est relié au déversoir par un barrage latéral de béton en contrefort d'une longueur de $986 \mathrm{~m}$.

Sur la rive gauche il a été édifié un barrage d'enrochement, qui est relié d'une part, au barrage principal et à l'autre extrémité, à un barrage de terre. Ces massifs d'une longueur totale de $4278 \mathrm{~m}$, sont crêtés à la côte 225 ; la hauteur maximale du barrage d'enrochement est de $70 \mathrm{~m}$.

$\mathrm{Au}$ point où la hauteur de ce barrage latéral atteint approximativement $30 \mathrm{~m}$, le massif passe du type "enrochement" au type "en terre".

\section{f) Déversoir}

Le déversoir est situé sur la rive droite. Les ouvrages de contrôle ont $390 \mathrm{~m}$ de largeur, avec la crête à la cote 200, et comprennent 14 vannes/secteur de $20 \mathrm{~m} \times 21,34 \mathrm{~m}$. Pour faciliter l'entretien, le déversoir est divisé en 3 parties, séparées par murs diviseurs. La capacité maximale de décharge du déversoir est de $62200 \mathrm{~m}^{3} / \mathrm{s}$ avec le déversoir a la cote $223 \mathrm{~m}$ (niveau maximal de crue).

\section{g) Prises d'eau}

Les prises d'eau pour les 18 groupes, se situent à la partie supérieure du barrage principal et de la structure de dérivation. Les conduites forcées dont le diamètre interne est de $10,50 \mathrm{~m}$, ont la partie intermédiaire fixée au parement aval du barrage. Chaque conduite forcée a près de $142 \mathrm{~m}$ de longueur totale jusqu'à l'entrée de la bâche spirale.

\section{h) Centrale}

La centrale est prévue pour l'installation de 18 groupes, à invervalles de $34 \mathrm{~m} ; 15$ de ces unités seront situées sur le chenal principal et les 3 autres sur le canal de dérivation.

Les dimensions totales de la centrale sont les suivantes :

- Longueur totale : $968 \mathrm{~m}$

- Hauteur maximale : $112 \mathrm{~m}$

- Largeur maximale : $99 \mathrm{~m}$.

Chaque groupe comprend une turbine Francis, d'une puissance nominale de $715 \mathrm{MW}$ entraînant un alternateur. Vitesse de rotation 90,9 à $92,3 \mathrm{t} / \mathrm{min}$., pour un débit de $698 \mathrm{~m}^{3} / \mathrm{s}$ et une hauteur de chute de $112,9 \mathrm{~m}$. Neuf des 18 alternateurs ont une puissance unitaire de 737 MVA sous $60 \mathrm{~Hz}$ et les neuf autres une puissance unitaire de 823,6 MVA sous $50 \mathrm{~Hz}$.

Le programme de construction porte sur une période de 8 ans, de 1975 à 1983, pour le génie civil.

Entre l'installation du premier groupe, et celle du $18^{\grave{e}}, 5$ ans s'écouleront encore. La centrale doit être totalement achevée en 1988.

Les structures de l'usine d'Itaipu achevées, comprendront les volumes suivants de béton :

- Barrage principal

$5160000 \mathrm{~m}^{3}$

- Structure de dérivation

$2140000 \mathrm{~m}^{3}$

- Barrage latéral droit

$770000 \mathrm{~m}^{3}$

- Déversoir

- Centrale

- Autres

$715000 \mathrm{~m}^{3}$

$2535000 \mathrm{~m}^{3}$

$500000 \mathrm{~m}^{3}$

Total $\quad \overline{11820000 \mathrm{~m}^{3}}$

Les valeurs maximales de production de béton jusqu'à ce jour (mars 1982) sont les suivantes :

- Par jour

$14986 \mathrm{~m}^{3}$

- Par période de 30 jours

$339000 \mathrm{~m}^{3}$

- Annuel

$3000000 \mathrm{~m}^{3}$

\section{i) Capacité énergétique}

La centrale hydroélectrique de Itaipu aura une chute brute de $120 \mathrm{~m}$, produira en moyenne $8000 \overline{\mathrm{MW}}$ (ou $70 \mathrm{TWh} / \mathrm{an}$ ) d'énergie garantie et son exploitation se fera un fil de l'eau, sans baisse sensible du niveau du réservoir.

La capacité de production de la centrale, représente environ $2.7 \%$ du potentiel total du bassin du Parana brésilien. De ce total de $8000 \overline{\mathrm{MW}}$, la moitié revient au Paraguai, mais sera utilisée par le Brésil pendant quelques années jusqu'au moment où le Paraguai en aura l'utilisation. La capacité énergétique de la centrale de Itaipu au moment de sa mise en service en 1983 représentera environ $50 \%$ de la consommation électrique du Brésil et près de $70 \%$ de la consommation électrique de la région du Sud-Est, mais sa capacité de production ne pourra satisfaire que pendant quelques 
années à la croissance de la demande électrique de la région.

\section{Conclusion}

Cette brève présentation montre la grande importance du bassin du Parana pour le développement du système électrique brésilien.

Il représente environ $28 \%$ du potentiel hydroélectrique total du pays et en 1990 près de $60 \%$ de l'énergie électrique utilisée.

Son aménagement planifié et programmé comprend la construction d'un nombre considérable de grands ouvrages. Ce programme de projets hydroélectriques a contribué de façon décisive à satisfaire à la demande d'énergie électrique et a permis par sa séquence conti- nuelle et planifiée des grands ouvrages, la consolidation de la technologie de génie civil, (construction, fabrication d'équipements et gérance dans le pays), concourant fortement au développement économique et institutionnel du pays.

On doit souligner l'importance pour le programme d'aménagement des ressources hydroélectriques, les études d'inventaire et de planification, faites de façon systématique dans les années 60 , et qui continuent de façon permanente.

L'aménagement des ressources hydroélectriques brésiliennes et en particulier celles du bassin du Parana peuvent être un exemple pour la conduite de programmes semblables, et servir de modèle à des pays dont les conditions et la situation économique sont identiques à celles au Brésil dans les années 1960 .

\title{
Discussion
}

\author{
Président : M. LONDE
}

M. le Président. - Merci, M. AMARANTE, pour votre remarquable présentation. Je dois vous dire que nous sommes surpris que vous ayez cru utile de vous excuser en ce qui concerne votre français, car il est merveilleux.

La discussion est ouverte. M. AMARANTE est prêt à répondre à vos questions. Et, si vous le permettez, je commencerai par en poser une, qui $m$ 'est venue à l'esprit pendant l'exposé.

Avez-vous, dans l'optimisation du projet de vos aménagements, pris en compte d'autres objectifs que la production de l'énergie, tels que la maîtrise des crues ou d'autres effets secondaires?

M. AMARANTE. -- L'objectif principal qui détermina la décision et sa justification économique fut la production d'énergie électrique, l'optimisation de l'opération étant uniquement examinée sous cet angle.

De multiples fonctions complémentaires ont bien étê considérées lors de la décision mais d'éventuels travaux d'aménagement pourront être envisagés ultérieurement.

Pour le bassin du Parana, il n'y a pas eu précisément d'études économiques sur l'opportunité, la faisabilité et la rentabilité des objectifs secondaires, la base du projet étant uniquement la production d'électricité.

M. le Président, - $\mathrm{J}$ e vous remercie de votre réponse. Qui demande la parole?

M. AUBERT. - Monsieur le Président, la question des buts secondaires, que vous avez posée à notre excellent conférencier, m'intéresse particulièrement.

Il y a deux ans, j'ai eu l'occasion de rencontrer à Rio le Général Cavalcanti, co-Président de la Société d'Itaipu. Je l'ai interrogé sur ses projets relatifs au franchissement du barrage par les bateaux. Il m'a répondu que la construction des écluses, dont le projet a été dressé, correspondait à une grosse dépense et qu'il n'avait pas l'intention d'ouvrir prochainement les chantiers correspondants. Il attendra pour le faire l'époque où il disposera des ressources financières que lui procureront la vente du courant électrique.

Je reconnais que la question de la navigation sort un peu du programme de ces journées, mais $j$ 'aimerais pouvoir m'en entretenir directement avec M. AMARANTE, pendant son actuel séjour à Paris. Je ne veux donc pas retenir plus longtemps, dans cette enceinte, l'attention d'auditeurs dont bien peu s'intéressent à la navigation.

(Protestation des représentants de la Cie nationale du Rhône)

M. COTILLON. - Je voudrais demander au Président AMARANTE si toutefois ma question n'est pas trop indiscrète, si le site d'Itaipu est un site bon marché ou, au contraire, un site cher, comparé aux sites d'amont qui viennent d'être équipés : Embarcaçâo, Itumbiara et Sâo Simâo ? Comment le coût du $\mathrm{kW}$ installé se place-t-il par rapport à celui des aménagements d'amont mentionnés ci-dessus ?

M. AMARANTE. - Il n'y a pas de différence sensible entre ces différentes installations ; c'est du même ordre de grandeur : environ 1000 dollars par $\mathrm{kW}$ installé. Pour les autres aménagements, le prix de revient de l'énergie devrait être de l'ordre de 25 cents le $\mathrm{kWh}$.

M. BERTRAND. - Le projet d'Itaipu est commun au Brésil et au Paraguay. Le financement est entièrement assuré par le Brésil, le Paraguay remboursant par la vente de l'électricité à venir, dont de toutes façons il n'a pas besoin, car elle dépasserait largement sa consommation nationale.

Est-ce de la part du Brésil un geste de générosité, ou est-ce que le choix du site imposait ce projet commun, et les relations diplomatiques cet aménagement financier?

M. AMARANTE. - En réalité, la chute d'Itaipu a été aménagée sur les $200 \mathrm{~km}$ de la partie internationale du cours du Parana, de sorte qu'il est normal que l'énergie produite sur cette partie du fleuve soit réellement partagée à raison de $50 \%$ pour le Brésil et $50 \%$ pour le Paraguay.

Il n'était pas possible pour le Brésil d'utiliser à lui seul toute l'énergie produite par cette usine ou il aurait fallu étudier un projet réduit n'en produisant que la moitié, mais c'eût été très difficile parce que les conditions du fleuve dans cette zone ne facilitent pas la construction de deux aménagements.

La décision de faire un projet commun a donc été quasiment une décision technique. Peut être les intérêts diplomatiques sontils également venus jouer en faveur de cette programmation. En réalité, ce choix est la meilleure solution.

Il n'y a pas de générosité de la part du Brésil, l'énergie d'Itaipu étant très économique ; le Brésil finance l'ensemble du 
projet parce que le site d'Itaipu est international et que la part paraguayenne sera remboursée en énergie.

M. MARINIER. - La zone du réservoir devait sans doute concerner des populations riveraines. Qu'advient-il de ces populations et combien de gens seront-ils inondés et déplacés ?

M. AMARANTE. - Il y en aura très peu. La surface inondable est très grande mais la population n'est pas considérable. Je n'ai pas le chiffre exact mais il en doit pas concerner plus de mille personnes.

Nous avons un autre projet au Nord-Est, la Palagino, où ce problème est beaucoup plus aigu. A Itaipu, le risque d'inondation éventuelle des villes et villages est relativement très faible.

M. CORDELLE. - Je voudrais poser deux questions à $M$. AMARANTE : la première concerne la navigation. La navigation existe sur le Parana à l'aval du confluent du Rio Iguaçu. J'ai cru voir aussi, sur les photos que vous nous avez montrées, que certains aménagements comportent des écluses. Y a-t-il de la navigation sur certaines parties du Parana et de ses principaux constituants?

Deuxième question : le rapprochement de la capacité d'Itaipu ( 30 milliards de $\mathrm{m}^{3}$, soit $30 \mathrm{~km}^{3}$ ) et du débit du fleuve montrent que la retenue sera remplie en 1 mois environ; quelles sont les mesures qui ont été prises pour "surveiller" la mise en eau du barrage alors qu'il est impossible d'interrompre cette mise en eau?

M. AMARANTE. - La navigation fluviale n'est pas importante sur cette partie du Parana et elle ne justifie pas un abaissement important du plan d'eau pendant la construction du barrage. Les transports par voie d'eau, dans la région d'Itaipu, ont été remplacés par des transports par camions. Dans le projet global, la réalisation des écluses a été prévie et pourra être envisagée ultérieurement à n'importe quel moment.

Le remplissage va se faire entre 15 et 20 jours environ au mois d'octobre de cette année. Le Parana sera entièrement coupé et toute l'alimentation en aval du barrage sera assurée par l'évacuateur de crue d'Iguaçu qui a une capacité de l'ordre de $5000 \mathrm{~m}^{3} / \mathrm{s}$. Ce sera une opération très intéressante et très complexe pour la gestion simultanée des évacuateurs de crue des diverses usines qui continueront à produire de l'électricité Le temps de remplissage étant très court, les lâchures d'eau en aval seront faites pour maintenir le fleuve au niveau minimum qui a été accepté par l'Argentine pour cette opération.

M. COURIER. - Je voudrais poser une question sur les groupes d'Itaipu : est-ce que les 18 turbines d'Itaipu sont identiques pour les deux fréquences de 50 et $60 \mathrm{~Hz}$ ? (même dessin des roues)?

M. AMARANTE. - Elles sont pratiquement les mêmes, mais elles ont été dessinées spécialement, les unes pour le $50 \mathrm{~Hz}$ et les autres pour le $60 \mathrm{~Hz}$, la vitesse de rotation des turbines étant légèrement différente.

M. Le Président. - J'ai cru comprendre que, dans l'avenir, vous passeriez de 50 à $60 \mathrm{~Hz}$ pour la fourniture au Brésil.

M. AMARANTE. - Les alternateurs fourniront toujours du $50 \mathrm{~Hz}$ pour les besoins du Brésil. Une ligne de transport en courant continu permettra la transformation du $60 \mathrm{~Hz}$ en $50 \mathrm{~Hz}$.

M. ROBERT. - Ma question porte sur la capacité des évacuateurs de crue. La valeur de $62000 \mathrm{~m} 3 / \mathrm{s}$ indiquée est impressionnante. La fixation de cette valeur, même lorsqu'on dispose d'une vaste information hydrologique est un problème difficile et essentiel pour la sécurité. Comment ce problème a-t-il été traité pour Itaipu?
M. AMARANTE. - Itaipu se situe à l'aval d'un très grand bassin qui est entièrement au Brésil. Lors des études de débit, on a construit un modèle réduit du Parana avec ses affluents et l'évaluation de la crue maximale a été faite par simulation des précipitations atmosphériques sur le bassin versant et les cours d'eau, compte-tenu de l'influence des réservoirs et de l'accélération de la crue du fait de la surface plane du réservoir. La crue maximale atteinte dans ce modèle était de l'ordre de 70 à $72000 \mathrm{~m}^{3} / \mathrm{s}$, mais il y avait un amortissement à l'étage du déversoir qui limitait à $68000 \mathrm{~m}^{3} / \mathrm{s}$ le débit sur le modèle. On peut suppoer ce maximum de précipitations de crue comme possible. Vous êtes peut-être impressionné par les $62000 \mathrm{~m}^{3} / \mathrm{s}$, mais je peux vous indiquer qu'en Amazonie, à Tucurui, un évacuateur de crue aura une capacité de $104000 \mathrm{~m}^{3} / \mathrm{s}$. C'est beaucoup.

M. le Président. - Il faut peut-être ajouter qu'il y a un facteur favorable de la prévisibilité de la crue ; dans un tel bassin, on doit pouvoir, dans une certaine mesure, ouvrir les organes d'évacuation et améliorer l'effet d'amortissement.

M. AMARANTE. - En ce qui concerne la pluviométrie, nous disposions d'une centaine d'années d'observations sur le bassin versant.

M. Le Président. - Nous allons arrêter les débats sur cette première communication, en remerciant $M$. AMARANTE à la fois pour la façon brillante avec laquelle il a présenté son rapport et pour la façon dont il a pu répondre à toutes nos questions.

Nous interrompons la séance pour vingt minutes.

La séance est interrompue de $10 \mathrm{~h} 20$ à $10 \mathrm{~h} \mathrm{40)}$.

M. Le Président. - Nous reprenons nos travaux avec le deuxième exposé de la séance de ce matin, qui sera présenté par M. MARINIER.

M. MARINIER est Vice-Président, pour l'ingénierie et le Développement, à la Société d'Energie de la Baie James. Je voudrais rappeler que M. MARINIER a été stagiaire en France au titre de l'ASTEF en 1959 et qu'il a eu à l'époque des contacts avec l'EDF et avec SOGREAH. Il a eu une carrière d'ingénieur de bureau d'études, notamment avec la grande Société SNC de Montréal. Il a participé au projet de Manicouagan et à bien d'autres. Actuellement, M. Gilles MARINIER est Vice-Président du Comité National Canadien des Grands Barrages et il aura une fonction importante à remplir à Rio de Janeiro au Congrès International de Mai 1983 étant Rapporteur Général de la Question 52 qui traite des problèmes de sécurité des barrages en exploitation.

Je donne la parole à M. MARINIER, qui va vous parler des aménagements de la Baie James.

Je voudrais ajouter une précision sur le programme. L'exposé de M. MARINIER sera suivi d'un premier film, puis la discussion sera ouverte, et ensuite, un deuxième film sera présenté.

M. MARINIER. - Merci, Monsieur le Président.

Mesdames, Messicurs, c'est avec un vif plaisir que j'ai accepté l'invitation de la Société Hydrotechnique de France pour venir vous exposer les grandes lignes de l'équipement hydroélectrique de la Baie James. Plusieurs d'entre vous connaissent certainement déjà l'Hydro-Québec ; quant à la Société d'Energie de la Baie James, elle a été créée en 1971 afin de prendre en charge l'aménagement hydroélectrique des rivières de ce territoire. En 1978 la Société d'Energie de la Baie James est devenue une filiale à part entière d'Hydro-Québec et elle fonctionne depuis sous mandat pour la réalisation des études d'ingénierie, la programmation et la gérance des aménagements de la Baie James. 